\title{
Stereotactic ablative body radiotherapy as a bridge to liver transplantation for hepatocellular carcinoma: preliminary results of Baskent University experience
}

\author{
Guler Yavas ${ }^{1}$, Ebru H. Ayvazoglu Soy ${ }^{2}$, Mehmet Coskun ${ }^{3}$, Cem Onal ${ }^{1}$, Fatih Boyvat ${ }^{3}$, Mehmet Haberal ${ }^{2}$
}

\footnotetext{
${ }^{1}$ Department of Radiation Oncology, Baskent University, Ankara, Turkey

${ }^{2}$ Department of Surgery, Baskent University, Ankara, Turkey

${ }^{3}$ Department of Radiology, Baskent University, Ankara, Turkey
}

Background: Hepatocellular carcinoma (HCC) is the most common primary liver tumor. The only curative treatment options remain to be liver transplantation and resection. However approximately $20 \%-30 \%$ of the patients have substantial disease progression while still awaiting transplantation. Herein, we report our initial experience on stereotactic ablative body radiotherapy (SABR) as a bridge to liver transplantation for HCC.

Methods: Nine lesions of seven patients received SABR as a bridge treatment to transplantation. All the patients underwent radiofrequency ablation, transcatheter arterial chemoembolization or hepatic resection prior to SABR. Radiographic response was based on magnetic resonance imaging (MRI) evaluation at one month after SABR.

Results: The median age of the patients was 65 years (range, 63-71 years). The median dose was 45 Gy (range, $45-54$ Gy) in 3 fractions. The median diameter of the lesions was $18 \mathrm{~mm}$ (range, $16-30 \mathrm{~mm}$ ). All the patients received SABR for single lesion except for two patients. No patient developed gastrointestinal toxicity or radiation-induced liver disease (RILD). Acute toxicity was minimal; all patients completed the full course. RILD was evaluated using liver enzyme, bilirubin, and albumin levels; no significant change supervened after the completion of SABR, and a month after SABR. A month after the SABR response rates were evaluated with MRI. In two lesions complete responses obtained. There were two partial responses and two stable diseases.

Conclusions: Herein, we report initial results Baskent University's experience with the safety and efficacy of SABR as another treatment option for bridging therapy. SABR is an effective, safe and tolerable treatment option for bridging therapy. However, we obtained early response to SABR, the exact response rates will be encountered at least 3 months after SABR. Therefore, our finding should be clarified with further prospective studies with long-term follow-up period. 